\title{
ON A TRANSFORMATION OF BILATERAL SERIES WITH APPLICATIONS
}

\author{
GEORGE E. ANDREWS ${ }^{1}$
}

\begin{abstract}
This paper is devoted to the study of a simple transformation of bilateral series. Formulae for basic bilateral hypergeometric series and generalizations of theorems on mock theta functions are proved.
\end{abstract}

1. Introduction. The object of this paper is to study the following very elementary result.

Transformation Lemma. Subject to suitable convergence conditions, if $c_{n}=\sum_{m=0}^{\infty} a_{m+n} b_{m}$, then

$$
\sum_{m=0}^{\infty} b_{m} \sum_{n=-\infty}^{\infty} a_{n}=\sum_{n=-\infty}^{\infty} c_{n}
$$

The proof of this result is quite simple

$$
\sum_{m=0}^{\infty} b_{m} \sum_{n=-\infty}^{\infty} a_{n}=\sum_{m=0}^{\infty} b_{m} \sum_{n=-\infty}^{\infty} a_{n+m}=\sum_{n=-\infty}^{\infty} \sum_{m=0}^{\infty} b_{m} a_{n+m}=\sum_{n=-\infty}^{\infty} c_{n} .
$$

In the cases we shall treat all series will be absolutely convergent, in particular for $\sum_{m} \sum_{n} \alpha_{m n}=\sum_{n} \sum_{m} \alpha_{m n}$ it is sufficient to have both double series absolutely convergent.

This simple transformation has been used in one form or another by several authors [2], [3], [6], [11]. Our object here is to systematically explore several special cases of this result. In particular, it was hoped that it might be possible to sum the basic bilateral hypergeometric series ${ }_{2} \psi_{2}$. Such a summation formula was originally sought by Bailey [4]; Agarwal and Verma [1] state that they were motivated to develop the theory of bi-basic hypergeometric series in the hope of finding such a formula. An incorrect summation of the ${ }_{2} \psi_{2}$ is given in [10]; this may be seen by substituting $b \rightarrow b t, c \rightarrow c t$, $d \rightarrow d t, e \rightarrow e t$ in [10, Equation (1)] and verifying that the series satisfies a first order $q$-difference equation in $t$ which is inconsistent with a first order $q$-difference equation satisfied by the product. Unfor-

Received by the editors November 10, 1969.

AMS Subject Classifications. Primary 1048, 3320.

Key Words and Phrases. Basic bilateral hypergeometric series, Ramanujan, mock theta functions.

1 Partially supported by National Science Foundation grant GP-8075. 
tunately, the most we could obtain in this direction is enunciated in Theorem 4.

2. Applications. In this section we refer to the Transformation Lemma as TL. We utilize the notation

$$
\begin{aligned}
(A ; q)_{\infty}= & (A)_{\infty}=\prod_{j=0}^{\infty}\left(1-A q^{j}\right), \quad(A ; q)_{n}=(A)_{n}=(A)_{\infty} /\left(A q^{n}\right)_{\infty}, \\
& { }_{r} \phi_{s}\left[\begin{array}{l}
A_{1}, \cdots, A_{r} ; t, q \\
B_{1}, \cdots, B_{s}
\end{array}\right]=\sum_{n=0}^{\infty} \frac{\left(A_{1}\right)_{n} \cdots\left(A_{r}\right)_{n} t^{n}}{\left(B_{1}\right)_{n} \cdots\left(B_{s}\right)_{n}(q)_{n}}, \\
& { }_{r} \psi_{s}\left[\begin{array}{l}
A_{1}, \cdots, A_{r} ; t, q \\
B_{1}, \cdots, B_{s}
\end{array}\right]=\sum_{n=-\infty}^{\infty} \frac{\left(A_{1}\right)_{n} \cdots\left(A_{r}\right)_{n}}{\left(B_{1}\right)_{n} \cdots\left(B_{s}\right)_{n}} t^{n},
\end{aligned}
$$

and

$$
\Pi\left[\begin{array}{l}
A_{1}, \cdots, A_{r} ; q \\
B_{1}, \cdots, B_{s}
\end{array}\right]=\prod_{i=1}^{r}\left(A_{i}\right)_{\infty} / \prod_{j=1}^{s}\left(B_{j}\right)_{\infty} .
$$

THEOREM 1.

$$
{ }_{1} \psi_{1}\left[\begin{array}{c}
\alpha ; t, q \\
\gamma
\end{array}\right]=\prod\left[\begin{array}{ccc}
\gamma / \alpha \beta t, & \gamma / \alpha, & \gamma / \beta ; q \\
\gamma / \alpha t, & \gamma, & \gamma / \alpha \beta
\end{array}\right]{ }_{1} \psi_{1}\left[\begin{array}{c}
\alpha ; t, q \\
\gamma / \beta
\end{array}\right] .
$$

Proof. In the TL, take

$$
\begin{aligned}
a_{n} & =\frac{(\alpha)_{n} t^{n}}{(\gamma)_{n}}, \quad b_{n}=\frac{(\beta)_{n}(\gamma / \alpha \beta t)^{n}}{(q)_{n}}, \\
c_{n} & =\prod\left[\begin{array}{cc}
\gamma / \alpha, & \gamma / \beta ; q \\
\gamma, & \gamma / \alpha \beta
\end{array}\right] \frac{(\alpha)_{n} t^{n}}{(\gamma / \beta)_{n}} .
\end{aligned}
$$

Then $c_{n}=\sum_{m=0}^{\infty} a_{m+n} b_{m}$ is merely a restatement of the $q$-analog of Gauss's theorem [9, p. 97, Equation (3.3.2.5)]. Hence by the TL, we obtain Theorem 1.

Corollary (Ramanujan [5, p. 194, Equation (1.3)]).

$$
{ }_{1} \psi_{1}\left[\begin{array}{c}
\alpha ; t, q \\
\gamma
\end{array}\right]=\Pi\left[\begin{array}{cccc}
q / \alpha t, & \gamma / \alpha, & q, & \alpha t ; q \\
\gamma / \alpha t, & \gamma, & q / \alpha, & t ;
\end{array}\right] .
$$

Proof. Put $\beta=\gamma / q$, and utilize

$$
{ }_{1} \psi_{1}\left[\begin{array}{c}
\alpha ; t, q \\
q
\end{array}\right]={ }_{1} \phi_{0}[\alpha ; t, q]=(\alpha t)_{\infty} /(t)_{\infty}
$$

by $[9$, p. 92, Equation (3.2.2.11)]. 
THEOREM 2. If

$$
h(z)=\left(-z q^{2} ; q^{4}\right)_{\infty}\left\{\sum_{n=0}^{\infty} q^{4 n^{2}} z^{2 n} /\left(-z q^{2} ; q^{4}\right)_{n}+\sum_{n=1}^{\infty} q^{2 n^{2}} z^{-n}\left(-z^{-1} q^{2} ; q^{4}\right)_{n}\right\},
$$

then

$$
h(z)+z q h\left(z q^{2}\right)=\frac{\left(q^{2} ; q^{2}\right)_{\infty}\left(-z q ; q^{2}\right)_{\infty}\left(-z^{-1} q ; q^{2}\right)_{\infty}}{\left(-q^{2} ; q^{2}\right)_{\infty}\left(q ; q^{5}\right)_{\infty}\left(q^{4} ; q^{5}\right)_{\infty}} .
$$

Proof. Let $b_{m}=q^{m^{2}} /\left(q^{4} ; q^{4}\right)_{m}, a_{m}=q^{m^{2}} z^{m}, c_{n}=q^{n^{2}} z^{n}\left(-z q^{2 n+2} ; q^{4}\right)_{\infty}$. Then $\sum_{m=0}^{\infty} a_{m+n} b_{m}=c_{n}$ is merely an elementary identity of Euler [9, p. 93, Equation (3.2.2.18)], while

$$
\sum_{m=0}^{\infty} \frac{q^{m^{2}}}{\left(q^{4} ; q^{4}\right)_{m}}=\left\{\left(q ; q^{5}\right)_{\infty}\left(q^{4} ; q^{5}\right)_{\infty}\left(-q^{2} ; q^{2}\right)_{\infty}\right\}^{-1},
$$

by $[8$, p. 154 , Equation (20)], and

$$
\sum_{m=-\infty}^{\infty} a_{m}=\left(q^{2} ; q^{2}\right)_{\infty}\left(-z q ; q^{2}\right)_{\infty}\left(-z^{-1} q ; q^{2}\right)_{\infty}
$$

is merely Jacobi's identity [9, p. 86, Equation (3.1.12)] which is actually a limiting case of the corollary of Theorem 1 . Finally by breaking $\sum_{n=-\infty}^{\infty} c_{n}$ into even and odd terms, we obtain

$$
h(z)+z q h\left(z q^{2}\right)=\sum_{n=-\infty}^{\infty} c_{n}
$$

which yields Theorem 2 .

Theorem 2 implies a result concerning Ramanujan's mock theta functions, since

$$
\begin{aligned}
h(1) & =\left(-q^{3} ; q^{4}\right)_{\infty}\left(F_{0}\left(q^{2}\right)+\varphi_{0}\left(q^{2}\right)-1\right), \\
h\left(q^{2}\right) & =\left(-q^{4} ; q^{4}\right)_{\infty}\left(f_{1}\left(q^{4}\right)+2 \psi_{1}\left(q^{4}\right)\right),
\end{aligned}
$$

where $F_{0}(q), \varphi_{0}(q), f_{1}(q)$, and $\psi_{1}(q)$ are mock theta functions [11, p. 278]. A result similar to Theorem 2 may be obtained by utilizing $b_{m}=q^{m^{2}+2 m} /\left(q^{4} ; q^{4}\right)_{m}, a_{m}=q^{m^{2}} z^{m} ; \sum b_{m}$ now becomes an infinite product by $[8$, p. 153 , Equation (16)].

THEOREM 3. If

$$
g(z)=(-z)_{\infty}\left\{\sum_{n=0}^{\infty} \frac{z^{2 n} q^{n^{2}-n}}{(-z ; q)_{n}}+\sum_{n=1}^{\infty} z^{-n} q^{1 / 2 n(n+1)}\left(-z^{-1} q\right)_{n}\right\},
$$

then 


$$
1 / 2\{g(z)+g(-z)\}=\frac{\left(q^{2} ; q^{2}\right)_{\infty}\left(-z^{2} ; q^{2}\right)_{\infty}\left(-z^{-2} q^{2} ; q^{2}\right)_{\infty}}{\left(q ; q^{2}\right)_{\infty}\left(q^{4} ; q^{20}\right)_{\infty}\left(q^{16} ; q^{20}\right)_{\infty}} .
$$

Proof. Here we set $b_{m}=q^{m^{2}} /(q)_{2 m}, a_{m}=q^{m^{2}-m} z^{2 m}, c_{m}=1 / 2 z^{2 m} q^{m^{2}-m}\left\{\left(-z q^{m} ; q\right)_{\infty}+\left(z q^{m} ; q\right)_{\infty}\right\}$. Then

$$
\begin{aligned}
\sum_{m=0}^{\infty} a_{m+n} b_{m} & =z^{2 n} q^{n^{2}-n} \sum_{m=0}^{\infty} \frac{q^{2 m 2+2 m n-m} z^{2 m}}{(q)_{2 m}} \\
& =1 / 2 z^{2 n} q^{n^{2}-n} \sum_{m=0}^{\infty} \frac{q^{1 / 2 m(m-1)+m n} z^{m}}{(q)_{m}}\left(1+(-1)^{m}\right) \\
& =c_{n},
\end{aligned}
$$

where the last line follows again from an identity of Euler [9, p. 93, Equation (3.22.18)]. It is now easily verified that $1 / 2\{g(z)+g(-z)\}$ $=\sum_{n=-\infty}^{\infty} c_{n}$. Finally

$$
\sum_{m=0}^{\infty} b_{m}=\left\{\left(q ; q^{2}\right)_{\infty}\left(q^{4} ; q^{20}\right)_{\infty}\left(q^{16} ; q^{20}\right)_{\infty}\right\}^{-1},
$$

by $[8$, p. 160 , Equation (79)], and

$$
\sum_{n=-\infty}^{\infty} a_{n}=\left(q^{2} ; q^{2}\right)_{\infty}\left(-z^{2} ; q^{2}\right)_{\infty}\left(-z^{-2} q^{2} ; q^{2}\right)_{\infty}
$$

by $[9$, p. 86, Equation (3.1.12)].

Just as with Theorem 2, Theorem 3 may also be related to Ramanujan's mock theta functions, and a result similar to Theorem 3 may be obtained by setting $b_{m}=q^{m^{2}+2 m} /(q)_{2 m+1}, a_{m}=q^{m^{2}-m} z^{2 m}$ and utilizing [8, p. 162, Equation (96)].

Our final result may be considered a reduction theorem for the general ${ }_{2} \psi_{2}$ in that we obtain an expansion of the general ${ }_{2} \psi_{2}$ in a series in which ${ }_{2} \psi_{2}$ 's with one vanishing parameter appear.

Theorem 4.

$$
\begin{aligned}
{ }_{2} \psi_{2}\left[\begin{array}{c}
a_{1}, a_{3} ; t, q \\
b_{1}, b_{2}
\end{array}\right]= & \prod\left[\begin{array}{c}
b_{1} b_{2} / a_{1} a_{3} t, b_{2} / a_{3} ; q \\
b_{2} ;
\end{array}\right] \\
& \cdot \sum_{m=0}^{\infty} \frac{\left(b_{1} / a_{1}\right)_{m}\left(a_{3}\right)_{m}\left(b_{2} / a_{3}\right)^{m}}{(q)_{m}\left(b_{1}\right)_{m}}{ }_{2} \psi_{2}\left[\begin{array}{c}
a_{1}, a_{3} q^{m} ; t, q \\
b_{1} q^{m}, 0
\end{array}\right] .
\end{aligned}
$$


Proof. We let

$$
\begin{gathered}
a_{m}=\frac{\left(a_{1}\right)_{m}\left(a_{3}\right)_{m} l^{m}}{\left(b_{1}\right)_{m}\left(b_{2}\right)_{m}}, \quad b_{m}=\frac{(-1)^{m} q^{1 / 2 m(m-1)}\left(b_{1} b_{2} / a_{1} a_{3} t\right)^{m}}{(q)_{m}}, \\
c_{n}=\frac{t^{n}\left(a_{1}\right)_{n}\left(b_{2} / a_{3}\right)_{\infty}}{\left(b_{2}\right)_{\infty}} \sum_{m=0}^{\infty} \frac{\left(b_{1} / a_{1}\right)_{m}\left(a_{3}\right)_{m+n}\left(b_{2} / a_{3}\right)^{m}}{(q)_{m}\left(b_{1}\right)_{m+n}} .
\end{gathered}
$$

Then $\sum_{m=0}^{\infty} a_{m+n} b_{m}=c_{n}$ follows directly from the identity

$$
\begin{aligned}
\lim _{a_{2} \rightarrow \infty}{ }_{3} \phi_{2} & {\left[\begin{array}{c}
a_{1}, a_{2}, a_{3} ; b_{1} b_{2} / a_{1} a_{2} a_{3}, q \\
b_{1}, b_{2}
\end{array}\right] } \\
= & \lim _{a_{2} \rightarrow \infty} \Pi\left[\begin{array}{c}
b_{2} / a_{3}, b_{1} b_{2} / a_{1} a_{2} ; q \\
b_{2}, b_{1} b_{2} / a_{1} a_{2} a_{3}
\end{array}\right]{ }_{3} \phi_{2}\left[\begin{array}{c}
b_{1} / a_{1}, b_{1} / a_{2}, a_{3} ; b_{2} / a_{3}, q \\
b_{1}, b_{1} b_{2} / a_{1} a_{2}
\end{array}\right]
\end{aligned}
$$

due to Sears [7, p. 174, Equation (10.1)].

Theorem 4 now follows directly from the TL.

It should be obvious from the preceding that many bilateral theorems may be obtained utilizing the TL. We have tried here to exhibit a few of the most interesting applications.

\section{REFERENCES}

1. R. P. Agarwal and Arun Verma, Generalized basic hypergeometric series with unconnected bases, Proc. Cambridge Philos. Soc. 63 (1967), 727-734. MR 35 \#3090.

2. G. E. Andrews, A simple proof of Jacobi's triple product identity, Proc. Amer. Math. Soc. 16 (1965), 333-334. MR $30 \# 1952$.

3. - On Ramanujan's summation of ${ }_{1} \psi_{1}(a ; b ; z)$, Proc. Amer. Math. Soc. 22 (1969), 552-553.

4. W. N. Bailey, On the basic bilateral hypergeometric series ${ }_{2} \psi_{2}$, Quart. J. Math. Oxford Ser. (2) 1 (1950), 194-198. MR 12, 178.

5. G. H. Hardy, Ramanujan. Twelve lectures on subjects suggested by his life and work, Cambridge Univ. Press, New York, 1940. MR 3, 71.

6. P. Kesava Menon, On Ramanujan's continued fraction and related identities, J. London Math. Soc. 40 (1965), 49-54. MR 30 \#3057.

7. D. B. Sears, On the transformation theory of basic hypergeometric functions, Proc. London Math. Soc. (2) 53 (1951), 158-180. MR 13, 33.

8. L. J. Slater, Further identities of the Rogers-Ramanujan type, Proc. London Math. Soc. (2) 54 (1952), 147-167. MR 14, 138.

9. - Generalized hypergeometric functions, Cambridge Univ. Press, Cambridge, 1966. MR $34 \# 1570$.

10. M. Upadhyay, Certain transformations and summation formulae for basic bilateral hypergeometric series ${ }_{2} \psi_{2}$, Amer. Math. Monthly 75 (1968), 171-172. MR 37 \#1654.

11. G. N. Watson, The mock theta functions. II, Proc. London Math. Soc. (2) 42 (1937), 274-304. 